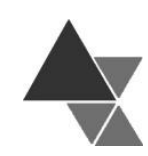

\title{
Condição de (In)Segurança Alimentar e fatores associados de famílias com crianças menores de cinco anos de idade do Estado do Maranhão
}

\author{
Naruna Pereira Rocha1, Sophia Cornbluth Szarfarc ${ }^{2}$, Pedro Israel Cabral de Lira ${ }^{3}$, \\ Leopoldina Augusta Souza Sequeira ${ }^{3}$, Victor Nogueira da Cruz Silveira ${ }^{4}$ e Maria Tereza \\ Borges Araújo Frota ${ }^{4}$
}

Este trabalho teve por objetivo analisar a condição de (In)Segurança Alimentar e fatores associados em famílias com crianças menores de cinco anos de idade no Estado do Maranhão. Trata-se de um estudo transversal de base populacional, descritivo e analítico, composto por 978 famílias atendidas pela Estratégia de Saúde da Família, no estado do Maranhão, em 2010. A situação de insegurança alimentar foi avaliada pelo questionário socioeconômico e demográfico, por meio de visitas domiciliares, além da Escala Brasileira de Insegurança Alimentar. Foram encontradas $70,5 \%$ das famílias em situação de insegurança alimentar. Correlação positiva entre a insegurança alimentar e os fatores demográficos e sociais, tais como localização do domicílio, coexistência de três ou mais crianças com idade inferior a cinco anos, baixa renda familiar, escolaridade e classe social foram encontrados. Concluiu-se que os resultados deste trabalho revelam uma expressiva frequência de familias com crianças menores de cinco anos sobrevivendo em situação de vulnerabilidade e confirmam os resultados divulgados por pesquisas nacionais.

Palavras-chave: insegurança alimentar e nutricional, família, crianças, mulheres.

\section{Condition of Food (In)Security and associated factors of families with children under five years old of the State of Maranhão}

This study aimed to analyze the condition of Food (In)Security and factors related to families with children under five years of age in the State of Maranhão. This is a cross-sectional study of population, descriptive and analytical basis, consisting of 978 families assisted by the Family Health Strategy in the state of Maranhão in 2010. The situation of food insecurity was assessed by socioeconomic and demographic questionnaire during home visits beyond Brazilian Food Insecurity Scale. 70.5\% of households were in food insecure. Positive correlation between food insecurity and demographic and social factors, such as location of the home, coexistence of three or more children under the age of five, low family income, education and social class were found. These results show a

1 Universidade Federal de Viçosa, MG, Brasil.

2 Departamento de Nutrição da Faculdade de Saúde Pública da Universidade de São Paulo, SP, Brasil.

${ }^{3}$ Departamento de Nutrição da Faculdade de Saúde Pública da Universidade de São Paulo, SP, Brasil.

${ }^{4}$ Universidade Federal do Maranhão, MA, Brasil. Endereço para correspondência: Maria Tereza Borges Araújo Frota - Av. dos Portugueses, no 1.966, Vila Bacanga, CEP: 65065-

545, São Luis, MA. Telefone: (98) 99974-5289. E-mail: mariaterezafrota@gmail.com 
significant rate of families with children under five years surviving in a vulnerable situation and confirm the results reported by national surveys.

Keywords: food insecurity, family, children, women.

\section{INTRODUÇÃO}

Em muitos países e regiões em desenvolvimento, particularmente em relação aos grupos sociais e familias de baixas condições socioeconômicas, o acesso regular e permanente a uma cesta básica de produtos suficiente e adequada para uma boa condição de saúde e nutrição mostra-se problemático, representando uma condição de insegurança ${ }^{[1]}$. Apesar da tendência de queda na situação de insegurança alimentar, esta se mantém elevada, especialmente em regiões historicamente vulneráveis, como o Nordeste[2].

O Direito Humano à Alimentação Adequada (DHAA) está previsto originalmente na Declaração Universal dos Direitos Humanos de 1988, sendo discutido no contexto da promoção do direito a um padrão adequado de vida. O mesmo direito é reafirmado no Pacto Internacional dos Direitos Econômicos, Sociais e Culturais (PIDESC), que entrou em vigor em 03 de janeiro de 1976, além de estar incluído em várias leis vigentes no Estado brasileiro, como na Lei Orgânica de Segurança Alimentar e Nutricional - LOSAN (Lei no 11.346, de 15 de julho de 2006) e na lei que reinstituiu o Conselho Nacional de Segurança Alimentar e Nutricional em 2006[3,4].

De acordo com documento aprovado na II Conferência Nacional de Segurança Alimentar e Nutricional (SAN), e incorporado na LOSAN, SAN é definida como a realização do direito de todos ao acesso regular e permanente a alimentos de qualidade, em quantidade suficiente, sem comprometer o acesso a outras necessidades essenciais, tendo como base práticas alimentares promotoras de saúde, que respeitem a diversidade cultural e que sejam social, econômica e ambientalmente sustentáveis[5,6].

Segundo o Programa das Nações Unidas para o Desenvolvimento (PNUD), no ano de 2013, o Índice de Desenvolvimento Humano (IDH) do Brasil era de 0,727 . No mesmo ano, o Maranhão encontrava-se em situação desfavorável em relação aos outros estados do Brasil, ocupando a penúltima posição, com Índice de Desenvolvimento Humano Municipal igual a 0,757[7].

No Brasil, 35,5\% das famílias destacam que a quantidade de alimento consumido é normalmente insuficiente ou eventualmente insuficiente. No Nordeste, os níveis de insatisfação com a quantidade de alimentos consumidos pelas famílias são altos ${ }^{[8]}$. Todos os estados da região registram proporções inferiores de segurança alimentar em relação à média nacional. No Maranhão, menos da metade dos domicílios tem alimentação saudável e em quantidade suficiente assegurada[9].

A situação de vulnerabilidade alimentar da população maranhense foi confirmada pela PNAD (2014), que revelou que $22,6 \%$ da população sobreviviam em situação de insegurança alimentar ${ }^{[10]}$.

Considerando a importância de se avaliar a situação de (in)segurança alimentar e os possíveis fatores de risco para essa situação, este estudo objetiva avaliar a prevalência de insegurança alimentar em famílias com crianças menores de cinco anos no estado do Maranhão, localizado na região nordeste do Brasil.

\section{MATERIAL E MÉTODOS}

Trata-se de um estudo transversal, descritivo e analítico, de base populacional, desenvolvido em 21 municípios do estado do Maranhão e na capital do estado (São Luís), randomizado de acordo com as macrorregiões a que pertenciam. A amostra foi calculada para ser representativa para o estado como um todo e para a capital. A população contemplou famílias atendidas pela Estratégia de Saúde da Família (ESF), com crianças de 6 meses a 59 meses de idade, residentes no estado do Maranhão.

Os dados foram obtidos no ano de 2010, entre os meses de julho a setembro, por meio de entrevistas domiciliares, com adoção de questionário semi- 
estruturado, pré-codificado e pré-testado em um estudo piloto no qual foram coletadas informações sobre a família, tais como: local de residência (urbana ou rural), composição familiar, condições socioeconômicas, demográficas, educação, renda, ocupação dos membros da família, número de refeições, acesso a programas de governo e bens de consumo.

Para a classificação socioeconômica das famílias foi utilizado o Critério de Classificação Econômica Brasil (CCEB) ${ }^{[11]}$ e para medir o nível de insegurança alimentar das famílias, foi aplicada a Escala Brasileira de Insegurança Alimentar (EBIA).

Os dados foram digitados em dupla entrada no Programa Epi Info versão 6.04 e transportados para planilha do Microsoft Office Excel 2007, para a elaboração das análises (frequência simples, média e cálculo dos Intervalos de Confiança de 95\% (IC95\%). Posteriormente, utilizou-se o programa Statistical Package for Social Sciences - SPSS (versão 12.01) para realização de regressão múltipla pelo método de Poisson. Adotou-se o nível de significância de $p<0,05$.

O trabalho foi aprovado pelos Comitês de Ética em Pesquisa com Seres Humanos da Universidade Federal do Maranhão (no 060/10) e da Faculdade de Saúde Pública da Universidade de São Paulo (no 2191/10). Todos os participantes do estudo concordaram e assinaram o Termo de Consentimento Livre e Esclarecido.

\section{RESULTADOS}

Nas 978 famílias investigadas predominou o sexo masculino como chefe da família $(69,1 \%)$, apontado por ser o principal ou único mantenedor da casa, além da referência como o responsável por decisões no âmbito do núcleo familiar. De acordo com a autodeterminação da cor do entrevistado, predominaram os que se declararam não brancos $(88,7 \%)$ e quanto à escolaridade do chefe da família, prevaleceu o tempo inferior a cinco anos de estudo $(44,4 \%)$.
Sobreviviam com uma renda per capita menor que 0,25 salário mínimo (SM) 54,6\% das famílias e em apenas $16,3 \%$ delas a renda foi superior a meio SM. No que se refere à classe econômica, predominaram as classes C $(29,3 \%)$, D $(38,3 \%)$ e E $(29,2 \%)$. Em quase metade das famílias $(48,1 \%)$, conviviam no mesmo domicílio de quatro a cinco pessoas. As residências com seis ou mais moradores representaram $31,6 \%$ da amostra. Estavam cadastradas e recebiam algum benefício do governo no momento da entrevista, 67,9\% das famílias, sendo o Programa Bolsa Família (PBF) o mais presente $(65,4 \%)$ (Tabela 1$)$. 
Tabela 1. Distribuição de famílias com crianças menores de cinco anos de idade, segundo variáveis socioeconômicas e demográficas, Maranhão, 2010

\begin{tabular}{|c|c|c|}
\hline VARIÁVEIS & $\mathrm{n}$ & $\%$ \\
\hline \multicolumn{3}{|l|}{ Sexo do chefe de família } \\
\hline Feminino & 302 & 30,9 \\
\hline Masculino & 676 & 69,1 \\
\hline \multicolumn{3}{|l|}{$\mathrm{Na}$ opinião do entrevistado - cor } \\
\hline Branca & 111 & 11,3 \\
\hline Não Branca & 867 & 88,7 \\
\hline \multicolumn{3}{|l|}{ Anos de estudo do chefe de família } \\
\hline Até 4 anos & 434 & 44,4 \\
\hline $5-8$ anos & 281 & 28,7 \\
\hline 9 ou mais anos & 263 & 26,9 \\
\hline \multicolumn{3}{|l|}{ Número de pessoas que moram na casa } \\
\hline 1 a 3 & 199 & 20,3 \\
\hline 4 a 5 & 470 & 48,1 \\
\hline$\geq 6$ & 309 & 31,6 \\
\hline \multicolumn{3}{|l|}{ Renda per capita (em salários mínimos): } \\
\hline$<0,25$ & 534 & 54,6 \\
\hline $0,25-0,49$ & 285 & 29,1 \\
\hline$\geq 0,50$ & 159 & 16,3 \\
\hline \multicolumn{3}{|l|}{ Classificação socioeconômica (CCEB) } \\
\hline A e B & 30 & 3,1 \\
\hline $\mathrm{C}$ & 287 & 29,3 \\
\hline $\mathrm{D}$ & 375 & 38,3 \\
\hline $\mathrm{E}$ & 286 & 29,2 \\
\hline \multicolumn{3}{|l|}{ Beneficiário de Programas Sociais } \\
\hline Sim & 664 & 67,9 \\
\hline Não & 314 & 32,1 \\
\hline \multicolumn{3}{|l|}{ Beneficiário do Programa Bolsa Família } \\
\hline Sim & 640 & 65,4 \\
\hline Não & 338 & 34,6 \\
\hline Total & 978 & 100,0 \\
\hline
\end{tabular}

Tabela 2. Prevalência de (In)Segurança Alimentar em domicílios com crianças menores de cinco anos. Maranhão, 2010

\begin{tabular}{lccc}
\hline SITUAÇÃO DE SEGURANÇA ALIMENTAR & $\mathbf{n}$ & $\mathbf{0}$ & IC 95\% \\
\hline Segurança alimentar & 288 & 29,4 & 26,9 a 32,8 \\
Insegurança alimentar leve & 315 & 32,2 & 29,1 a 35,0 \\
Insegurança alimentar moderada & 222 & 22,7 & 20,1 a 25,5 \\
Insegurança alimentar grave & 153 & 15,6 & 13,3 a 17,9 \\
\hline Total & $\mathbf{9 7 8}$ & $\mathbf{1 0 0 , 0}$ & \\
\hline
\end{tabular}

Verificou-se que 29,4\% das famílias avaliadas viviam em situação de segurança alimentar e que 70,5\% passavam por algum grau de insegurança alimentar e nutricional. A insegurança leve foi a mais frequente, acometendo 32,2\% das familias (Tabela 2).
Todas as macrorregiões do estado apresentaram a insegurança alimentar leve (maior prevalência), com exceção da macrorregião de Pinheiro em que prevaleceu a insegurança alimentar grave (31\%). Observa-se que o maior valor de SA $(35,9 \%)$ foi encontrado na capital São Luís, que foi descrita isoladamente e não integra de nenhuma das macrorregiões (Gráfico 1). 
Gráfico 1. Situação de Segurança Alimentar segundo as Macrorregiões e a Capital do Estado. Maranhão, 2010

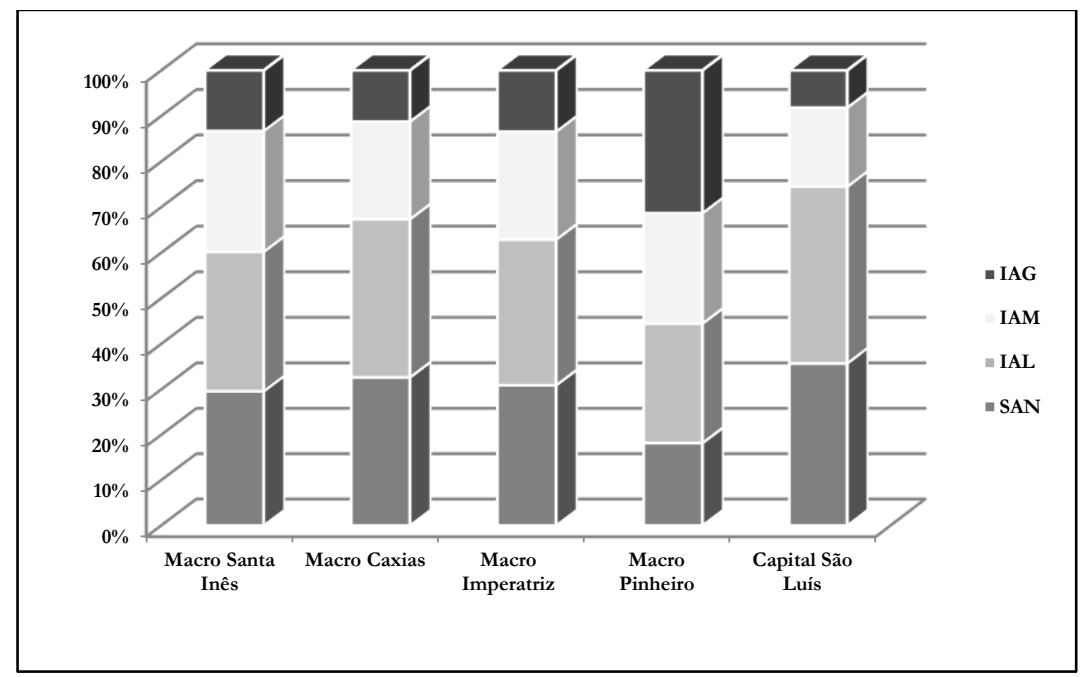

Neste estudo, morar no interior $(p=0,229)$ ou em área rural $(p=0,25)$ não se associou estatisticamente ao risco de estar em situação de insegurança alimentar. As famílias que apresentavam rendimento per capita inferior a 0,25 SM e entre 0,25 e 0,49 SM aumentavam em 1,94 vezes e em 1,46 vezes, respectivamente, a chance de se expor com algum nível de insegurança alimentar. As famílias que pertenciam às classes $\mathrm{D}$ e $\mathrm{E}$ apresentaram maiores risco de estar em situação de insegurança alimentar e nutricional $(p<0,004$ e $p<0,001$, respectivamente), quando comparadas às famílias das classes A, B e C.

O fato do domicilio apresentar esgotamento sanitário inadequado, contribuiu para que as famílias com crianças menores de cinco anos tivessem 1,37 vezes mais chances de apresentarem insegurança alimentar e nutricional $(p<0,001)$.

Observa-se que tanto a escolaridade da mãe, quanto do chefe da família, bem como a situação do emprego, número de pessoas no domicílio e número de crianças menores de cinco anos de idade estiveram associados positivamente à situação de insegurança alimentar vivida pelas famílias (Tabela 4).

A renda per capita esteve associada significativamente à situação de insegurança alimentar das famílias. A média da renda mensal da população exposta à insegurança alimentar grave era de $\mathrm{R} \$ 281,00$ $(p<0,001)$, muito abaixo das famílias consideradas com $\mathrm{SA}(\mathrm{R} \$ 760,00)$.

Analisando a distribuição da população atendida pela ESF, segundo a IA e ser ou não beneficiário do Bolsa Família, a proporção de famílias que apresentaram qualquer grau de insegurança é significativamente menor entre aquelas que recebiam benefício $(p<0,001)$. A análise multivariada revelou que as familias que dispunham de rendimentos per capita inferior a $1 / 4$ de salário mínimo tinham 1,9 vezes mais chances de apresentar algum grau de IA quando comparadas a aquelas que recebiam mais de 0,5 salário mínimo ( $p<0,001$; IC: $1,51-2,51)$. 
Tabela 4. Situação de Segurança Alimentar das famílias com crianças menores de cinco anos segundo as condições socioeconômicas e demográficas. Maranhão, 2010

\begin{tabular}{|c|c|c|c|}
\hline \multicolumn{2}{|c|}{ VARIÁVEIS } & \multicolumn{2}{|c|}{ SEGURANÇA ALIMENTAR } \\
\hline & $\mathbf{R P}$ & IC $(95 \%)$ & $p$ \\
\hline \multicolumn{4}{|c|}{ Localização do Domicílio } \\
\hline Interior & 1,13 & $0,93-1,36$ & 0,229 \\
\hline Capital & 1,00 & & \\
\hline \multicolumn{4}{|c|}{ Área do domicílio } \\
\hline Rural & 1,10 & $0,94-1,28$ & 0,250 \\
\hline Urbano & 1,00 & & \\
\hline \multicolumn{4}{|c|}{$\begin{array}{l}\text { Renda per capita (em salários } \\
\text { mínimos) }\end{array}$} \\
\hline$<0,25$ & 1,94 & $1,51-2,51$ & $<0,001$ \\
\hline $0,25-0,49$ & 1,46 & $1,10-1,93$ & $<0,008$ \\
\hline$>0,5$ & 1,00 & & \\
\hline \multicolumn{4}{|c|}{ Classe Econômica } \\
\hline $\mathrm{D}$ & 1,33 & $1,10-1,61$ & $<0,004$ \\
\hline $\mathrm{E}$ & 1,61 & $1,32-1,96$ & $<0,001$ \\
\hline $\mathrm{A}, \mathrm{B}, \mathrm{C}$ & 1,00 & & \\
\hline \multicolumn{4}{|l|}{ Cor da pele } \\
\hline Não Branca & 1,08 & $0,85-1,37$ & 0,549 \\
\hline Branca & 1,00 & & \\
\hline \multicolumn{4}{|c|}{ Escolaridade da Mãe (em anos) } \\
\hline 0 a 4 & 1,29 & $1,06-1,55$ & 0,009 \\
\hline 4 a 8 & 1,23 & $1,04-1,47$ & 0,019 \\
\hline $9 \mathrm{ou}+$ & 1,00 & & \\
\hline \multicolumn{4}{|c|}{ Escolaridade Chefe (em anos) } \\
\hline 0 a 4 & 1,27 & $1,05-1,54$ & 0,014 \\
\hline 4 a 8 & 1,20 & $0,97-1,48$ & 0,014 \\
\hline $9 \mathrm{ou}+$ & 1,00 & & \\
\hline \multicolumn{4}{|c|}{ Emprego do Chefe } \\
\hline Informal & 1,25 & $1,05-1,48$ & 0,012 \\
\hline Formal & 1,00 & & \\
\hline \multicolumn{4}{|c|}{ Número de pessoas no domicílio } \\
\hline$>6$ & 1,25 & $1,00-1,55$ & 0,047 \\
\hline 4 e 5 & 1,13 & $0,92-1,39$ & 0,242 \\
\hline$\leq 3$ & 1,00 & & \\
\hline \multicolumn{4}{|c|}{$\begin{array}{l}\text { Número de crianças < de } 5 \text { anos no } \\
\text { domicílio }\end{array}$} \\
\hline$\geq 3$ & 1,40 & $1,09-1,81$ & 0,009 \\
\hline 2 & 1,20 & $1,02-1,42$ & 0,027 \\
\hline 1 & 1,00 & & \\
\hline \multicolumn{4}{|c|}{ Realização de lanches } \\
\hline Não & 1,23 & $1,04-1,45$ & 0,014 \\
\hline Sim & 1,00 & & \\
\hline \multicolumn{4}{|c|}{ Tratamento da água de beber } \\
\hline Não tratada & 1,13 & $0,97-1,31$ & 0,110 \\
\hline Tratada & 1,00 & & \\
\hline \multicolumn{4}{|c|}{ Esgotamento Sanitário } \\
\hline Inadequado & 1,37 & $1,18-1,60$ & $<0,001$ \\
\hline Adequado & 1,00 & & \\
\hline
\end{tabular}

\section{DISCUSSÃO}

A prevalência de insegurança alimentar apresentada pelas famílias com crianças menores de cinco anos de idade nesta pesquisa foi similar ao valor descrito pela PNAD (2014) ${ }^{[10]}$ para o estado do Maranhão (70,9\%). Em 2006, a Pesquisa Nacional de Demografia e Saúde encontrou uma prevalência de $54,6 \%$ de insegurança alimentar para toda a região nordeste[12]. Os valores reafirmam a gravidade da 
situação de insegurança alimentar e a vulnerabilidade vivida pelas famílias atendidas, em todo o estado, pelo programa Estratégia de Saúde da Família.

Alguns estudos realizados na região nordeste também destacam elevadas prevalências de insegurança alimentar como a pesquisa de Oliveira et al. (2010)[1], realizada no município de Gameleira (PE), que revelou a existência de $75 \%$ das famílias com crianças menores de cinco anos de idade que sobreviviam em situação de insegurança alimentar moderada e grave. No município de São João do Tigre (PE), a prevalência de IA chegava a $87,3 \%$ da população de mulheres e crianças ${ }^{[13]}$. Na cidade de Maceió (AL), dentre os beneficiários do Programa Bolsa Família, 79,8\% dos domicílios com crianças e adolescentes encontravam-se em insegurança alimentar moderada e grave ${ }^{[14]}$.

Os melhores indicadores de segurança alimentar apresentados pela capital podem ser atribuídos possivelmente, à melhor estabilidade de emprego e renda, acesso aos alimentos, informação, bens e serviços, fatores que podem contribuem para a melhoria da situação alimentar das famílias.

Dentre as macrorregiões, a de Pinheiro apresentou os menores valores de segurança alimentar. No entanto, estabelecer as causas desses resultados tem como fator limitante a falta de dados e de pesquisas regionais publicados que permitam comparações. Os municípios que integram a Macrorregião de Pinheiro compartilhavam de valores elevados de incidência de pobreza no ano da pesquisa, em que o menor valor encontrado foi de $56,37 \%$ (Turiaçú) e o maior $62,89 \%$ (Governador Nunes Freire). Em relação ao Índice de Gini, que mede o grau de desigualdade (com base nos rendimentos), quanto mais próximo de um, maior a desigualdade, Governador Nunes Freire $(0,33)$ e Bacuri $(0,38)$ apresentavam os maiores valores ${ }^{[15]}$. Essas características dos municípios pertencentes a Macrorregião de Pinheiro pode ter influenciado diretamente nos resultados encontrados.

As famílias que sobreviviam com menos de meio salário mínimo per capita tiveram quase duas vezes mais riscos de apresentarem insegurança alimentar que as famílias que tinham rendimento per capita superior. Vários estudos comprovam que famílias com baixos rendimentos têm maiores chances para a ocorrência de insegurança alimentar, visto que a economia brasileira é mercantil e para ter acesso aos alimentos é necessário ter renda. Por isso quanto menores os rendimentos, menores as chances de acesso a alimentos em quantidade e qualidade satisfatórias ${ }^{[16,17]}$.

Uma das grandes causas da falta de acesso aos alimentos é o baixo nível de renda enfrentada pelas familias brasileiras ${ }^{[18]}$. Barros et al. (2001) ${ }^{[19]}$ relatam que a desigualdade de renda consiste em um dos principais problemas enfrentados por expressiva parcela da população brasileira e acabam por ser um dos principais condicionantes da pobreza.

A renda familiar, portanto, consiste em um dos fatores chaves para a retirada dessa população da situação de insegurança alimentar. O aumento da renda, seja por acesso a programas assistenciais ou por melhoria da educação e consequentemente da ocupação de melhores empregos, elevaria a condição socioeconômica e a posição social das famílias. Logo, ainda que não fosse suficiente para a eliminação da IA, poderia retirar a família da situação de insegurança grave[20].

A escolaridade do chefe das famílias e dos moradores contribui para a situação de segurança

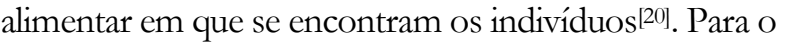
Brasil, urbano ou rural, quanto maior o nível de escolaridade dos moradores menor a prevalência de insegurança moderada ou grave, visto que a formação educacional proporciona maiores chances de empregabilidade formal e melhor remuneração ${ }^{[10]}$.

Algumas pesquisas destacam a influência das condições econômicas e sociais, como classe social, escolaridade dos pais, condição de habitação e emprego, como variáveis fortemente associadas ao consumo familiar[21,22,23].

No que se refere ao tipo de esgotamento sanitário presente nas moradias, Panigassi et al. (2008) ${ }^{[17]}$ relatam que a ausência de rede de esgoto nos domicílios pode elevar a probabilidade de insegurança alimentar moderada e grave em 5,3 vezes e de insegurança alimentar leve em 1,9 vezes.

Mais da metade das famílias avaliadas recebiam algum benefício do governo e em sua maior parte era proveniente do Programa Bolsa Família. Esses auxílios estão relacionados à melhoria das condições sociais e da qualidade de vida, sendo essenciais para identificar populações vulneráveis e traçar estratégias que visem à 
redução das desigualdades sociais ${ }^{24]}$. Hoffmann (2008)[20], em análise dos determinantes da insegurança alimentar no Brasil, relata que a baixa renda é o mais importante determinante da IA, o que ressalta a importância da transferência de renda como o PBF.

Estudo que analisou a influência do PBF sobre a SA nos domicílios brasileiros demonstrou que a participação nesse programa eleva em 7,4 pontos percentuais (p.p.) a probabilidade de um domicilio estar em condição de SA. Estimou, ainda, que o PBF eleva em cerca de 11 p.p. as chances de um domicílio sair da condição de levemente seguro para a condição de seguro, mas não parece afetar a condição de domicílios em IA moderada e grave, pelo menos não a ponto de se tornarem seguros. Ainda de acordo com o autor, o efeito do PBF recai mais fortemente sobre os domicílios próximos a linha da pobreza ${ }^{[25]}$.

Este estudo restringiu-se às famílias com crianças menores de cinco anos atendidas pela Estratégia de Saúde da Família, que em sua maioria constitui-se de uma população de baixo poder aquisitivo. A situação de insegurança alimentar é uma questão de saúde pública e é maior entre os domicílios particulares com pelo menos um morador menor de 18 anos de idade em relação aos domicílios onde só habitam adultos, visto que familias com muitas crianças são grupo de risco, pois há a necessidade de dividir a renda familiar, já pequena, em maior quantidade de pessoas, refletindo em maior pobreza ${ }^{[10]}$.

Os resultados aqui apresentados indicam um problema de grande magnitude, pois tratam-se de familias que possuem em sua constituição pelo menos uma criança menor de cinco anos, o que torna ainda mais grave a situação, por se tratar de um grupo populacional vulnerável nos quais as consequências nutricionais são imediatas e graves ${ }^{[16]}$.

Contrastando com os péssimos indicadores sociais e de segurança alimentar encontrados nesta pesquisa e em outras referidas, o Maranhão é um estado notadamente diversificado e rico, com uma economia voltada principalmente para o comércio exterior [26]. Além disso, sua bacia hidrográfica é privilegiada, com rios perenes e estação de chuvas regulares, o que o diferencia dos demais estados do Nordeste que padecem de longos períodos de estiagem e seca[27]. Essas características geográficas mais favoráveis em relação aos outros estados do Nordeste poderiam estar associadas a melhores condições de vida e menores valores de insegurança alimentar.

No entanto, sucessivas gestões públicas que não colocam os grupos vulneráveis no centro de suas preocupações e não incorporam a dimensão do direito humano nas políticas públicas fizeram com que, apesar de todas as riquezas que possui, o Maranhão seja o estado com o maior percentual da população socialmente excluída, com Índice de Exclusão Social (IES) igual a 59,56\%, sendo maior que os demais estados do Nordeste[28].

Para algumas variáveis avaliadas não foram encontradas associações estatísticas, provavelmente pela homogeneidade da amostra em relação às classes sociais a que pertenciam e por apresentarem o perfil da população usuária do Sistema Único de Saúde. Por se tratar de um estudo transversal, também não foi possível estabelecer a causalidade entre os fatores avaliados. Porém, este trabalho contribui para expor a realidade vivida e a situação alimentar de famílias com mulheres e crianças menores de cinco anos do estado do Maranhão.

É importante compreender que a situação de insegurança alimentar que o Maranhão apresenta é principalmente devido ao modelo de desenvolvimento econômico excludente que contribui para o aumento da concentração de renda e da desigualdade social que impactam negativamente sobre a qualidade de vida e condições de saúde da população. A implantação das políticas públicas de combate à fome e à pobreza e $\mathrm{o}$ cumprimento das que existem devem priorizar as populações vulneráveis, pois são imprescindíveis para a melhoria das condições sociais.

Este estudo contribui para o retrato da situação de SAN no Estado do Maranhão, caracterizando a realidade vivida pelas famílias com crianças menores de cinco anos atendidas pela Estratégia de Saúde da Família e que necessitam de ações emergenciais para a realização de um direito básico presente na Constituição Federal, o direito humano à alimentação adequada.

\section{CONCLUSÃO}

Os dados encontrados sobre a situação de insegurança alimentar no Maranhão confirmam os resultados divulgados pelas pesquisas realizadas no país 
e revelam um problema de grande magnitude que atinge famílias com crianças menores de cinco anos sobrevivendo em situação de insegurança alimentar.

As correlações entre a insegurança alimentar e os fatores demográficos e sociais, como renda familiar, classe social, escolaridade, número de moradores e presença de esgotamento sanitário indicam a necessidade de avançar nas formulações de propostas que integrem a população como um todo, além de iniciativas locais bem sucedidas que possibilitem a distribuição mais igualitária da renda, a erradicação da pobreza e a melhoria dos níveis de renda, fatores que influenciam diretamente na questão da alimentação e nutrição.

\section{REFERÊNCIAS}

[1] Oliveira JS, Lira PIC, Maia SR, Sequeira LAS, Amorim RCA, Batista Filho M. Insegurança alimentar e estado nutricional de crianças de Gameleira, zona da mata do Nordeste brasileiro. Rev. Bras. Saúde Mater. Infant. 2010;10(2):237-245.

[2] Instituto Brasileiro de Geografia e Estatística - IBGE. Pesquisa Suplementar de Segurança Alimentar. Rio de Janeiro: IBGE; 2014.

[3] Valente F, Franceschini T, Burity V. A exigibilidade do direito humano à alimentação adequada [Internet]. Brasília (DF): ABRANDH; 2007 [acesso em 01 mar 2017]. Disponível em: http://www.abrandh.org.br

[4] Brasil. Conselho Nacional de Segurança Alimentar e Nutricional - CONSEA. Lei no 11.346, de 15 de setembro de 2006. Cria o Sistema Nacional de Segurança Alimentar e Nutricional - SISAN com vistas em assegurar o direito humano à alimentação adequada e dá outras providências. Diário Oficial da União. 15 set 2006.

[5] Albuquerque MFM. A segurança alimentar e nutricional e o uso da abordagem de direitos humanos no desenho das políticas públicas para combater a fome e a pobreza. Rev. Nutr. 2009;22(6):895-903.

[6] Conselho Nacional de Segurança Alimentar e Nutricional. Conceitos: Segurança Alimentar e Nutricional e Soberania Alimentar. Brasilia (DF): CONSEA; 2017.

[7] Programa das Nações Unidas para o Desenvolvimento. Desenvolvimento Humano nas Macrorregiões Brasileiras. Brasilia (DF): PNUD; 2013.
[8] Instituto Brasileiro de Geografia e Estatística - IBGE. Pesquisa de Orçamentos Familiares: despesas, rendimentos e condições de vida. Rio de Janeiro: IBGE; 2010.

[9] Araújo MSS, Sousa SMPS, Nascimento TS. (In)Segurança Alimentar no Estado do Maranhão. Boletim do Grupo de Avaliação e Estudo da Pobreza e de Políticas Direcionadas à Pobreza-GAEPP - Universidade Federal do Maranhão. São Luis: GAEPP; 2014.

[10] Instituto Brasileiro de Geografia e Estatística - IBGE. Pesquisa Nacional por Amostra de Domicilios. Rio de Janeiro: PNAD; 2014.

[11] Associação Brasileira de Empresas de Pesquisa. Critério de classificação econômica Brasil. Rio de Janeiro: ABEP; 2015.

[12] Ministério da Saúde. Pesquisa Nacional de Demografia e Saúde da Criança e da Mulher - PNDS 2006: dimensões do processo reprodutivo e da saúde da criança. Brasilia (DF): Ministério da Saúde; 2009.

[13] Oliveira JS, Lira PIC, Veras ICL, Maia SR, Lemos MCC, Andrade SLLS, et al. Estado nutricional e insegurança alimentar de adolescentes e adultos em duas localidades de baixo índice de desenvolvimento humano. Rev. Nutr. 2009;22(4):453-465.

[14] Cabral MJ, Vieira KA, Sawaya AL, Florêncio TMMT. Perfil socioeconômico, nutricional e de ingestão alimentar de beneficiários do Programa Bolsa Família. Estud. Av. 2013;27(78):71-87.

[15] Instituto Brasileiro de Geografia e Estatística. Censo Demográfico: IBGE Cidades - 12 Recenseamento Geral do Brasil. Rio de Janeiro: IBGE; 2010.

[16] Oliveira JS, Lira PIC, Andrade SLLS, Sales AC, Maia SR, Batista Filho M. Insegurança Alimentar e estado nutricional de crianças de São João do Tigre, no semi-árido do Nordeste. Rev. Bras. Epidemiol. 2009;12(3):413-423.

[17] Panigassi G, Segall-Correa AM, Marin-León L, PérezEscamilla R, Maranha LK, et al. Insegurança alimentar intrafamiliar e perfil de consumo de alimentos. Rev. Nutr. 2008;21(Suppl):135s-144s.

[18] Belik W. Perspectivas para Segurança Alimentar e Nutricional no Brasil. Saúde Soc. 2003;12(1):12-20.

[19] Barros RP, Henriques R, Mendonça RSA. Estabilidade inaceitável: desigualdade e pobreza no Brasil. Rio de Janeiro: IPEA; 2001. 
[20] Hoffmann R. Determinantes da Insegurança Alimentar no Brasil: análise dos dados da PNAD 2004. Segur. Aliment. Nutr. 2008;15(1):49-61.

[21] Romani SAM, Lira PIC. Fatores determinantes do crescimento infantil. Rev. Bras. Saúde Mat. Infant. 2004;4(1):15-23.

[22] Olinto MTA, Victora CG, Barros FC, Tomasi E. Determinantes da desnutrição infantil em uma população de baixa renda: um modelo de análise hierarquizado. Cad. Saúde Pública. 1993;9(Suppl 1):14-27.

[23] Monteiro CA, Freitas ICM. Evolução de condicionantes socioeconômicos da saúde na infância na cidade de São Paulo (1984-1996). Rev. Saúde Pública. 2000;34(6 Suppl):8-12.

[24] Martins APB, Canella DS, Baraldi LG, Monteiro CA. Transferência de renda no Brasil e desfechos nutricionais: revisão sistemática. Rev. Saúde Pública. 2013;47(6):11591171.

[25] Camelo, TS. Alimentação e nutrição e saúde em programas de transferência de renda: evidências para o Programa Bolsa família. Revista Economia. 2009;10(4):685713.

[26] Instituto Maranhense de Estudos Socioeconômicos e Cartográficos - IMESC. Produto interno bruto do Estado do Maranhão: período de 2004-2008. São Luís: IMESC; 2010.

[27] Pires IE, Ferreira CA. Potencialidade do Nordeste do Brasil para Reflorestamento. Curitiba: EMBRAPA-URPFCS; 1982 (Circular Técnica, 6).

[28] Silva OP. A exclusão social no semiárido brasileiro [monografia]. Fortaleza: Universidade Federal do Ceará; 2010 . 